\title{
Valeurs critiques asymptotiques d'une fonction définissable dans une structure o-minimale
}

\author{
par D. D'Acunto (Le Bourget du Lac)
}

\begin{abstract}
We prove that the set of asymptotic critical values of a $C^{1}$ function definable in an o-minimal structure is finite, even if the structure is not polynomially bounded. As a consequence, the function is a locally trivial fibration over the complement of this set.
\end{abstract}

1. Introduction. On sait que, pour une fonction de classe $C^{1}$ à valeurs réelles, définie sur un ouvert $\Omega$ de $\mathbb{R}^{n}$, les seules valeurs critiques (notées $\left.K_{0}(f)\right)$ ne suffisent pas à caractériser les points de bifurcation; c'est-à-dire les valeurs pour lesquelles la topologie des fibres change (voir en particulier l'exemple 2 de la section 5 , où toutes les fibres sont connexes exceptée la fibre au-dessus de 0 , bien que la fonction n'a pas de valeurs critiques). Il faut donc étudier quel autre type de phénomène peut se produire, et en conséquence, introduire la notion de singularité à l'infini (ou plus généralement celle de singularité au bord de $\Omega$ ), ainsi que celle d'ensemble de valeurs critiques asymptotiques (noté $K_{\infty}(f)$, cf. déf. 3.1 et thm. 4.2). Dans le cas d'une fonction $f$ définie sur $\mathbb{R}^{n}$, l'ensemble $\mathbb{R} \backslash K_{\infty}(f)$ est l'ensemble des points qui vérifient la condition de Malgrange (cf. [11]). Il est également connu (cf. [12]) que, lorsque $f$ est de classe $C^{2}, f$ est une fibration triviale au-dessus de chaque composante connexe de $\mathbb{R} \backslash\left(K_{0}(f) \cup K_{\infty}(f)\right)$. En général, l'ensemble $K_{f}=K_{0}(f) \cup K_{\infty}(f)$ est très grand, et peut être égal à $\mathbb{R}$. Dans toute la suite on supposera que la fonction $f$ est définissable dans une structure o-minimale (cf. [2]).

Le théorème principal de cette note (thm. 3.4) montre que $K_{\infty}(f)$ est fini (pour la finitude de $K_{0}$ voir [8]). La démonstration s'inspire de [5] où un résultat analogue est montré, mais avec une définition de valeurs critiques asymptotiques qui ne permet pas d'utiliser le théorème de Palais

\footnotetext{
2000 Mathematics Subject Classification: Primary 03C64, 26A12; Secondary 32S15, $58 \mathrm{~K} 05$.

Key words and phrases: o-minimal structure, bifurcation set, asymptotic critical value.
} 
(cf. [10] et [12]) qui est une généralisation du célèbre théorème de fibration d'Ehresmann dans le cas de fonctions non propres. Dans la section 4 nous généralisons le résultat principal de la section 3 au cas où $f$ est définie sur un ouvert $\Omega \neq \mathbb{R}^{n}$. Nous construisons une métrique (définissable) riemannienne $\delta$ sur $\Omega$ et nous montrons la finitude des valeurs critiques asymptotiques relativement à $\delta$ (thm. 4.2). Puisque $(\Omega, \delta)$ est complet, on peut appliquer le théorème de Palais. A la fin de cette note, nous montrons que pour $f: \mathbb{R}^{n} \rightarrow \mathbb{R}$, on a $S_{f} \subset K_{\infty}(f)$, où $S_{f}$ est un ensemble définissable qui contient déjà les points de bifurcation (cf. [8]).

Par ailleurs, la section 2 rappelle les résultats nécessaires sur les structures o-minimales.

Remerciements. Je remercie particulièrement les Professeurs K. Kurdyka, P. Orro et D. Trotman pour leurs conseils et les discussions que nous avons eues pendant la préparation de cette note. Je remercie également G. Comte pour ses remarques avisées.

\section{Structures o-minimales sur le corps des réels}

DÉfinition 2.1. Soit $\mathcal{M}=\bigcup_{n \in \mathbb{N}} \mathcal{M}_{n}$ où chaque $\mathcal{M}_{n}$ est une famille d'ensembles de $\mathbb{R}^{n}$. On dit que $\mathcal{M}$ est une structure o-minimale sur $(\mathbb{R},+, \cdot,<)$ si les axiomes suivants sont vérifiés :

(i) Chaque $\mathcal{M}_{n}$ est une algèbre booléenne de sous-ensembles de $\mathbb{R}^{n}$, c'est-à-dire $\emptyset \in \mathcal{M}_{n}$, si $A, B \in \mathcal{M}_{n}$ alors $A \cup B \in \mathcal{M}_{n}, A \cap B \in \mathcal{M}_{n}$, et $\mathbb{R}^{n} \backslash A \in \mathcal{M}_{n}$.

(ii) Si $A \in \mathcal{M}_{n}$ et $B \in \mathcal{M}_{m}$, alors $A \times B \in \mathcal{M}_{n+m}$.

(iii) Si $A \in \mathcal{M}_{n+m}$ et $\Pi: \mathbb{R}^{n+m} \rightarrow \mathbb{R}^{n}$ est la projection naturelle sur les $n$ premières coordonnées, alors $\Pi(A) \in \mathcal{M}_{n}$.

(iv) Si $f, g_{1}, \ldots, g_{k} \in \mathbb{R}\left[X_{1}, \ldots, X_{n}\right]$, alors $\left\{x \in \mathbb{R}^{n}: f(x)=0, g_{i}(x)>0\right.$, $i=1, \ldots, k\} \in \mathcal{M}_{n}$.

(v) Tout ensemble définissable dans $\mathcal{M}_{1}$ est réunion finie d'intervalles et de points.

Etant donnée une structure o-minimale arbitraire $\mathcal{M}$, on dira qu'un ensemble $A$ est définissable dans $\mathcal{M}$ s'il existe un entier $n$ tel que $A \in \mathcal{M}_{n}$. On dira qu'une fonction $f: A \rightarrow \mathbb{R}^{m}$ est définissable dans $\mathcal{M}$ si son graphe est un ensemble définissable dans $\mathcal{M}$.

Dans toute la suite $\mathcal{M}$ est une structure o-minimale arbitrairement fixée, et définissable sous-entend définissable dans $\mathcal{M}$.

Dans cette partie, on donne uniquement les résultats utilisés pour la démonstration du théorème principal (théorème 3.4).

Lemme 2.2 (Théorème de Monotonicité). Soit $f:] a, b[\rightarrow \mathbb{R}$ une fonction définissable, où $-\infty \leq a<b \leq \infty$. Alors il existe des réels $a=a_{0}<a_{1}<$ 
$\ldots<a_{k}=b$ tels que $f$ est de classe $C^{1}$ sur chaque intervalle $] a_{i}, a_{i+1}[$. De plus $f^{\prime}$ est strictement monotone ou constante sur tout intervalle $] a_{i}, a_{i+1}[$.

Preuve. Voir [2] ou [3].

Lemme 2.3 (Lemme du petit chemin). Soit $A \subset \mathbb{R}^{n}$ un ensemble définissable, et $a \in \overline{A \backslash\{a\}}$. Alors il existe un arc $\gamma$ définissable où $\gamma:\left[0, \varepsilon\left[\rightarrow \mathbb{R}^{n}\right.\right.$ est de classe $C^{1}$ tel que $a=\gamma(0)$ et $\gamma(] 0, \varepsilon[) \subset A \backslash\{a\}$.

Preuve. Voir [1], [2] ou [3].

Corollaire 2.4 (Lemme du petit chemin à l'infini). Soit $F: A \rightarrow \mathbb{R}^{k}$ une application définissable sur un ensemble définissable non borné $A \subset \mathbb{R}^{n}$. On suppose de plus qu'il existe une suite $\left\{x_{p}\right\}_{p \in \mathbb{N}}$ de points de A telle que

$$
\lim _{p \rightarrow \infty}\left\|x_{p}\right\|=\infty, \quad \lim _{p \rightarrow \infty} F\left(x_{p}\right)=a .
$$

Alors il existe un arc $\gamma:\left[R, \infty\left[\rightarrow \mathbb{R}^{n}\right.\right.$, définissable et de classe $C^{1}$, vérifiant

$$
\forall r \in\left[R, \infty\left[, \quad \gamma(r) \in A, \quad \lim _{r \rightarrow \infty}\|\gamma(r)\|=\infty, \quad \lim _{r \rightarrow \infty} F(\gamma(r))=a .\right.\right.
$$

Preuve. La preuve de ce résultat se fait essentiellement par compactification définissable du graphe de l'application $F$, afin de se ramener aux conditions du lemme précédent. En effet, si on désigne par $\Gamma_{F}$ le graphe de l'application $F$ et $\sigma: x \mapsto x /\|x\|^{2}$ définie pour $x \neq 0$, alors l'application

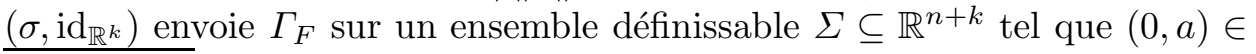
$\overline{\Sigma \backslash\{(0, a)\}}$. Il suffit alors d'appliquer le lemme précédent au point $(0, a)$ et à l'ensemble $\Sigma$, puis de décompactifier.

Pour la preuve du résultat principal, on a besoin de certaines propriétés des arcs définissables. Dans les lemmes suivants, on établit les notions de sécantes, et de tangentes à l'infini ainsi que l'existence et l'unicité de telles droites, étant donné un arc définissable de longueur infinie.

Lemme 2.5. Soit $\gamma:] R_{0}, \infty\left[\rightarrow \mathbb{R}^{n}\right.$ un arc définissable, de classe $C^{1}$, tel que $\lim _{r \rightarrow \infty}\|\gamma(r)\|=\infty$. Soit $S_{r}=\mathbb{R} \gamma(r)$ la droite vectorielle passant par le point $\gamma(r)$. De la même façon, on définit $T_{r}=\mathbb{R} \gamma^{\prime}(r)$. Alors $S_{\infty}=$ $\lim _{r \rightarrow \infty} S_{r}$ et $T_{\infty}=\lim _{r \rightarrow \infty} T_{r}$ existent.

Preuve. Si $\gamma$ est définissable, alors l'application $r \mapsto S_{r}$ est définissable, et à valeurs dans un compact. Il suffit de voir $\Gamma=\left\{S_{r}: r>R_{0}\right\}$ comme un arc définissable de classe $C^{1}$ inclus dans $\mathbb{P}^{n-1}(\mathbb{R})$. De plus sa frontière forme un ensemble définissable et de dimension nulle $\mathbb{P}^{n-1}(\mathbb{R})$, et la connexité de $\gamma$ implique l'existence de $S_{\infty}$. L'existence et l'unicité de $T_{\infty}$ se prouve de façon similaire.

Lemme 2.6. Soit $\gamma:] R_{0}, \infty\left[\rightarrow \mathbb{R}^{n}\right.$ un arc définissable, vérifiant les mêmes hypothèses que dans le lemme 2.5. Alors $T_{\infty}=S_{\infty}$. 
Preuve. Soit $u=\lim _{r \rightarrow \infty} \gamma(r) /\|\gamma(r)\|$ et $v=\lim _{r \rightarrow \infty} \gamma^{\prime}(r) /\left\|\gamma^{\prime}(r)\right\|$. Supposons au contraire $u \neq v$. Alors $(u, v)$ engendre dans $\mathbb{R}^{n}$ un plan $P$ de dimension 2. Ainsi, si $\Pi_{P}$ désigne la projection orthogonale sur $P$, et $\Pi_{P}(\gamma)$ l'image de $\gamma$, alors asymptotiquement $\Pi_{P}(\gamma)$ est le graphe d'une certaine fonction $g$ (c'est-à-dire $\Pi_{P}(\gamma)=\{(x, y) \in P: y=g(x)\}$ ) définissable, de signe constant et non nulle dans un système orthonormal de coordonnées où $S_{\infty}$ est la droite d'équation $y=0$. On aurait alors, vu que $u \neq v$, $\lim _{x \rightarrow \infty} g(x)=0$ et $\lim _{x \rightarrow \infty} g^{\prime}(x) \neq 0$, ce qui est impossible. Finalement $u=v$, et $T_{\infty}=S_{\infty}$

Conséquence 2.7. Pour $r$ suffisamment grand, l'angle entre $T_{r}$ et $S_{r}$ est arbitrairement proche de 0 et si $\|\gamma(r)\|=r$, alors $\left\|\gamma^{\prime}(r)\right\|$ est borné et on $a 1 \leq\left\|\gamma^{\prime}(r)\right\|<C$.

Preuve. En effet,

$$
2 r=\left(\|\gamma\|^{2}\right)^{\prime}(r)=\langle\gamma(r), \gamma(r)\rangle^{\prime}=2 r\left\|\gamma^{\prime}(r)\right\| \cos \left(\gamma(r), \gamma^{\prime}(r)\right),
$$

cest-à-dire $\left\|\gamma^{\prime}(r)\right\|=1 / \cos \left(\gamma(r), \gamma^{\prime}(r)\right)$. Et d'après le lemme 2.6 l'angle entre $T_{r}$ et $S_{r}$ tend vers 0 quand $r$ tend vers l'infini, soit $\cos \left(\gamma(r), \gamma^{\prime}(r)\right) \sim 1$. Et donc $\left\|\gamma^{\prime}(r)\right\|$ reste voisin de 1 pour $r$ assez grand.

Le résultat suivant est essentiel pour démontrer le lemme 3.3.

Lemme 2.8 (Lemme d'aile). Soient $V$ et $S$ deux ensembles définissables tels que $V \subset \bar{S} \backslash S$. Alors, il existe un ensemble définissable $A \subset S$ tel que $V=\bar{A} \cap(\bar{S} \backslash S)$.

Pre u ve. C'est un résultat classique dont la construction est identique à celle que l'on peut trouver dans un livre de géométrie semi-algébrique (voir par exemple [1]).

3. Valeurs critiques asymptotiques. Rappelons que si $t$ n'est pas une valeur critique, ni une valeur critique asymptotique (au sens de la définition ci-dessous), alors on dit que la condition de Malgrange est vérifiée en $t$, et il existe un voisinage $V$ de $t$ tel que $f: f^{-1}(V) \rightarrow V$ est une fibration. Pour les détails voir par exemple [10] et [11]. La section 4 énonce également une généralisation de ce résultat. Le résultat principal de ce papier (théorème 3.4) montre que pour une fonction définissable $C^{1}$, il n'y a qu'un nombre fini de points où la condition de Malgrange n'est pas vérifiée.

DÉfinition 3.1. Soit $f: \mathbb{R}^{n} \rightarrow \mathbb{R}$ une fonction différentiable. Soit $K_{\infty}(f)$ le sous-ensemble de $\mathbb{R}$ défini par

$$
\begin{aligned}
K_{\infty}(f)=\left\{c \in \mathbb{R}: \exists x_{p} \in \mathbb{R}^{n}(p \in \mathbb{N}), \lim _{p \rightarrow \infty}\left\|x_{p}\right\|=\infty,\right. \\
\left.\qquad \lim _{p \rightarrow \infty} f\left(x_{p}\right)=c, \lim _{p \rightarrow \infty}\left\|x_{p}\right\| \cdot\left\|\nabla f\left(x_{p}\right)\right\|=0\right\} .
\end{aligned}
$$


On dit que $c \in \mathbb{R}$ est une valeur critique asymptotique pour la fonction $f$ si $c \in K_{\infty}(f)$.

Remarque 3.2. Si $K_{0}(f)$ désigne l'ensemble des valeurs critiques de $f$, alors $K(f)=K_{\infty}(f) \cup K_{0}(f)$ n'est en général pas fermé pour une fonction de classe $C^{1}$ qui n'est pas propre. Si $f$ est définissable, alors les ensembles $K_{0}(f)$ et $K_{\infty}(f)$ sont fermés et définissables dans $\mathcal{M}_{1}$ (pour la définissabilité il suffit d'écrire $K(f)$ en langage de premier ordre et de procéder à l'élimination des quantificateurs). Ainsi $K_{\infty}(f)$ est au plus réunion finie d'intervalles et de points.

Lemme 3.3. Soit $f$ une fonction de classe $C^{1}$ définissable. Alors il existe $\varrho: \mathbb{R}_{+} \rightarrow \mathbb{R}_{+}$définissable vérifiant $\lim _{r \rightarrow \infty} \varrho(r) / r=\infty$ et telle que $K_{\infty}^{\varrho}(f)=K_{\infty}(f)$, ò̀

$$
\begin{aligned}
& K_{\infty}^{\varrho}(f)=\left\{c \in \mathbb{R}: \exists x_{p}\right. \in \mathbb{R}^{n}(p \in \mathbb{N}), \lim _{p \rightarrow \infty}\left\|x_{p}\right\|=\infty, \\
&\left.\qquad \lim _{p \rightarrow \infty} f\left(x_{p}\right)=c, \lim _{p \rightarrow \infty} \varrho\left(\left\|x_{p}\right\|\right)\left\|\nabla f\left(x_{p}\right)\right\|=0\right\} .
\end{aligned}
$$

Preuve. $K_{\infty}^{\varrho}(f) \subset K_{\infty}(f)$ est trivial. Il suffit de prouver l'inclusion dans le sens inverse. La démonstration reproduit dans les grandes lignes un résultat similaire de [7] dans le cadre semi-algébrique. Soit $k$ un entier positif. On identifie $\mathbb{R}^{k}$ avec $s_{k}\left(\mathbb{R}^{k}\right) \subset S^{k}$, où $s_{k}$ désigne l'inverse de la projection stéréographique. Soient $g$ et $\phi$ les fonctions définies sur $\mathbb{R}^{n}$ par $g(x)=\|x\| \cdot\|\nabla f(x)\|$ et $\phi(x)=(f(x), g(x))$. Soit $S=\operatorname{graph}(\phi) \subset$ $S^{n} \times S^{1} \times S^{1}$ et $V$ l'adhérence dans $S^{n} \times S^{1} \times S^{1}$ de $\{\infty\} \times K_{\infty}(f) \times\{0\}$. Il est clair que $V$ et $S$ vus commes des sous-ensembles de $S^{n} \times S^{1} \times S^{1}$ sont compacts et définissables, et que $V \subset \bar{S} \backslash S$. Et d'après le lemme 2.8, il existe $A \subset S$ tel que

$$
\bar{A} \cap\left(\{\infty\} \times S^{1} \times S^{1}\right)=V .
$$

Soit $\Gamma=\Pi(A)$ où $\Pi$ désigne la projection naturelle sur les $n$ premières coordonnés. Or (1) signifie simplement que $\{\infty\} \times\{y\} \times\{0\} \in V$ si et seulement s'il existe une suite de points $x_{p} \in \Gamma$ telle que $\left\|x_{p}\right\| \rightarrow \infty, f\left(x_{p}\right) \rightarrow$ $y$ et $g\left(x_{p}\right) \rightarrow 0$. Notons que la dernière condition est valide grace à la compactification initiale. Soit $\theta$ la fonction définie par

$$
\theta(r)=\sup _{x \in \Gamma \cap S^{n}(r)} r\|\nabla f(x)\| .
$$

La fonction $\theta$ est définissable et $\theta(r) \rightarrow 0$ quand $r \rightarrow \infty$. On pose alors $\varrho(r)=\left(\theta(r) / r^{2}\right)^{-1 / 2}$. Ainsi $\varrho(r) / r \rightarrow \infty$ et $(\varrho(r) / r) \theta(r) \rightarrow 0$. D'où

$$
\sup _{x \in \Gamma \cap S^{n}(r)} \varrho(r)\|\nabla f(x)\| \rightarrow 0
$$

et on obtient l'inclusion $K_{\infty}(f) \subset K_{\infty}^{\varrho}(f)$. 
ThÉorème 3.4. Soit $f: \mathbb{R}^{n} \rightarrow \mathbb{R}$ une fonction définissable et de classe $C^{1}$. Alors $K_{\infty}(f)$ est réunion finie de points.

Preuve. Compte tenu de la remarque précédente, on va montrer que $K_{\infty}(f)$ ne contient pas d'intervalle. La preuve se divise en deux parties distinctes, dont la première n'est valide que dans le cadre de structures ominimales polynomiallement bornées (i.e. pour toute fonction définissable $f: \mathbb{R} \rightarrow \mathbb{R}$, il existe $N \in \mathbb{N}$ tel que, asymptotiquement, $|f(x)|<x^{N}$ ). La seconde est valable dans toute structure o-minimale, mais utilise l'existence de décomposition L-régulière, qui est un résultat assez profond. Confronter ces deux démonstrations est révélateur de la différence entre les structures polynomiallement bornées (assez proches des ensembles semi-algébriques), et les structures non polynomiallement bornées qui contiennent nécessairement la fonction exponentielle (cf. [9]).

(A) La majeure partie de la preuve (jusqu'au moment ou il en est fait mention) se déroule sans restriction sur la nature de la structure.

Soit $\varrho$ comme dans le lemme précédent. On construit une fonction définissable $\varphi: \mathbb{R} \rightarrow \mathbb{R}_{+}$, nulle sur $K_{\infty}^{\varrho}(f)$ et strictement positive sur $\mathbb{R} \backslash K_{\infty}^{\varrho}(f)$, en posant

$$
\varphi(t)=\inf \left\{\varrho\left(\left\|x_{p}\right\|\right)\left\|\nabla f\left(x_{p}\right)\right\|: x_{p} \in \mathbb{R}^{n},\left\|x_{p}\right\| \rightarrow \infty, \lim _{p \rightarrow \infty} f\left(x_{p}\right)=t\right\} .
$$

Soit $c \in K_{\infty}^{\varrho}(f)$. Quitte à remplacer $f$ par $f-c$, on peut supposer que $0 \in K_{\infty}^{\varrho}(f)$. De plus, on peut supposer que $f^{-1}(t) \neq \emptyset$ pour $t>0$ et suffisamment proche de 0 . Dans le cas contraire, le théorème est trivial.

L'ensemble $K_{\infty}^{\varrho}(f)$ et la fonction $\varphi$ étant définissables, il suffit de montrer qu'il existe un réel $\delta>0$ tel que la fonction $\varphi$ est strictement positive sur l'intervalle $] 0, \delta[$. On suppose au contraire :

$(*) \quad$ il existe $\varepsilon>0$ tel que pour tout réel $t, 0<t<\varepsilon$, on a $\varphi(t)=0$.

On construit alors un autre ensemble définissable $\Sigma$, en posant

$$
\Sigma=\left\{x \in \mathbb{R}^{n}: \varrho(\|x\|)\|\nabla f(x)\|<f(x)\right\} .
$$

Ainsi, si $(*)$ est vérifié, il existe une suite $t_{n} \in \mathbb{R}$ telle que $\varphi\left(t_{p}\right)=0$ et $\lim _{p \rightarrow \infty} t_{p}=0$. Alors, il existe une suite $t_{p}^{\prime} \in \mathbb{R}$ vérifiant $\left|t_{p}^{\prime}-t_{p}\right|<t_{p} / 2$ et une suite $y_{p} \in f^{-1}\left(t_{p}{ }^{\prime}\right) \cap\left\{x \in \mathbb{R}^{n}:\|x\|>p\right\}$ telles que $\varrho\left(\left\|y_{p}\right\|\right)\left\|\nabla f\left(y_{p}\right)\right\|<$ $t_{p}^{\prime}$. Comme $\left(y_{p}, t_{p}^{\prime}\right) \in f_{\Sigma},\left\|y_{p}\right\| \rightarrow \infty$ et $t_{p}^{\prime} \rightarrow 0$ quand $p \rightarrow \infty$, il existe (d'après le lemme 2.5) un arc définissable $\widetilde{\gamma}$, de classe $C^{1}$, contenu dans $\mathbb{R}^{n} \times \mathbb{R}_{+}$, tel que, en paramétrant de sorte que $\|\gamma(r)\|=r$, on a $\widetilde{\gamma}(r)=$ $(\gamma(r),(f \circ \gamma)(r))$ pour tout $r>R$, et $\widetilde{\gamma}(] R, \infty[) \subset f_{\Sigma}$ pour un $R$ suffisamment grand.

De plus $\gamma$ vérifie les conditions suivantes :

$$
\lim _{r \rightarrow \infty}\|\gamma(r)\|=\infty \quad \text { et } \quad \lim _{r \rightarrow \infty}(f \circ \gamma(r))=0 .
$$


Maintenant, si $r \in] R, \infty[$, on pose $h(r)=(f \circ \gamma)(r)$, et on a

$$
\lim _{r \rightarrow \infty} h(r)=0 .
$$

Ensuite, comme $\left|h^{\prime}(r)\right|=\left|\left\langle\nabla f(\gamma(r)), \gamma^{\prime}(r)\right\rangle\right| \leq\|\nabla f(\gamma(r))\| \cdot\left\|\gamma^{\prime}(r)\right\|$, et $\gamma(r) \in \Sigma$, on obtient, grace à la conséquence 2.7,

$$
0<\left|h^{\prime}(r)\right| \leq \frac{h(r)}{\varrho(r)}\left\|\gamma^{\prime}(r)\right\| \leq C \frac{h(r)}{\varrho(r)} .
$$

Si la structure est polynomiallement bornée, alors il existe des uniques réels $\alpha, K>0$ tels que $\lim _{r \rightarrow \infty} \varrho(r) / r^{1+\alpha}=K$ (cf. [9]). La fonction $1 / \varrho$ est donc intégrable. Soit $r_{0}>R$ tel que pour tout $r>r_{0}$, l'inégalité précédente est vérifiée. Posons $u(r)=2 h\left(r_{0}\right)-h(r)$ pour $r>r_{0}$. Ainsi, on a $\lim _{r \rightarrow \infty} u(r)=2 h\left(r_{0}\right)$. Et l'inégalité $(2)$ implique

$$
0<u^{\prime}(r) \leq C \frac{u(r)}{\varrho(r)} \text {. }
$$

Ainsi en intégrant membre à membre l'inégalité (3) pour $s \in\left[r_{0}, r\right]$ et en utilisant le lemme de Gronwall, on obtient, pour des constantes $K_{1}>0$, dépendant de $K$ et de $C$, et $K_{2}>K_{1}$,

$$
u(r) \leq h\left(r_{0}\right) \exp \int_{r_{0}}^{\infty} \frac{K_{1}}{\varrho(s)} d s \leq h\left(r_{0}\right) \exp \left(\frac{K_{2}}{r_{0}^{\alpha}}\right)<\frac{3}{2} h\left(r_{0}\right),
$$

quitte à choisir $r_{0}$ suffisamment grand pour que $K_{2} / r_{0}^{\alpha}<\log \frac{3}{2}$. La fonction $u$ est donc majorée par un réel strictement plus petit que $2 h\left(r_{0}\right)$. Ceci est en contradiction avec $\lim _{r \rightarrow \infty} u(r)=2 h\left(r_{0}\right)$, et achève la démonstration.

Maintenant, si la structure n'est pas polynomiallement bornée, il se peut que la fonction $\varrho$ soit de la forme $\varrho(r)=r \log r$. Il est alors impossible d'utiliser un argument tel que le lemme de Gronwall. L'idée pour résoudre le problème est de définir, sur des sphères d'un rayon assez grand, une famille d'ensembles dont l'intersection des images par la fonction $f$ contient $K_{\infty}(f)$. Cette construction se fait par décomposition L-régulière d'une famille d'ensembles définissables.

(B) Soit $\Theta \subset \mathbb{R}^{n}$ l'ensemble définissable, défini par $\Theta=\left\{x \in \mathbb{R}^{n}\right.$ : $\|\nabla f(x)\|<1 / \varrho(\|x\|)\}$. On définit ensuite $\Theta_{r}=\Theta \cap S(r)$. D'après [4] (ou [6] pour une récente version valide dans une structure o-minimale quelconque), il existe une partition finie $\left\{L^{i}\right\}_{i \in I} \operatorname{de} \Theta=\bigcup_{r>0} \Theta_{r}$ telle que chaque $L^{i}$ est définissable et $L^{i}(r)=L^{i} \cap S(r)$ a la propriété de Whitney avec constante $M=M(n)$ et exposant 1. Cela signifie que la distance géodésique entre deux points $x$ et $y$ de $L^{i}(r)$ est inférieure à $M\|x-y\| \leq 2 M r$.

Soit $c$ une valeur critique asymptotique de la fonction $f$. On va encore montrer qu'il n'y a pas d'intervalle ouvert contenant $c$ et constitué uniquement de valeurs critiques asymptotiques. Remarquons d'abord que 
si $c \in K_{\infty}(f)$, alors $c \in \lim _{R \rightarrow \infty} \overline{\bigcup_{r>R} f\left(\Theta_{r}\right)}$. Par ailleurs, les $L^{i}$ étant en nombre fini, on déduit que $c \in \lim _{R \rightarrow \infty} \overline{\bigcup_{r>R} f(L(r))}$ où $L$ est l'un des $L^{i}$ de la décomposition. Soit $\gamma$ une géodésique contenue dans $L(r)$. Alors,

$$
\begin{aligned}
\operatorname{long}(f \circ \gamma) & =\int_{s_{1}}^{s_{2}}\left|(f \circ \gamma)^{\prime}\right|(s) d s \leq \int_{s_{1}}^{s_{2}}\left|\gamma^{\prime}(s)\right| \cdot\|\nabla f(\gamma(s))\| d s \\
& \leq \frac{1}{\varrho(r)} \operatorname{long}(\gamma) \leq \frac{2 M r}{\varrho(r)},
\end{aligned}
$$

où, par abus de notation, long désigne aussi bien la longueur d'un arc que celle d'un intervalle. Ainsi, $\operatorname{long}[f(L(r))] \leq 2 M r / \varrho(r) \rightarrow 0$ quand $r \rightarrow \infty$. Soit $[a(r), b(r)]$ l'intervalle $\overline{f(L(r))}$. Notons que les fonctions $a$ et $b$ sont définissables et par conséquent ont une limite commune (on désigne par $\alpha$ cette limite) en $\infty$, car $\lim _{r \rightarrow \infty}(a(r)-b(r))=0$. Rappelons que pour $r$ assez grand, $a$ et $b$ sont monotones. Soit

$$
I(R)=\overline{\bigcup_{r>R}[a(r), b(r)]} .
$$

Alors suivant la monotonie des fonctions $a$ et $b$, l'intervalle $I(R)$ se présente sous l'une des formes suivantes : $I(R)=[a(R), b(R)]$ ou $I(R)=[a(R), \alpha]$ ou $I(R)=[\alpha, b(R)]$. Et dans tous les cas $\lim _{R \rightarrow \infty} I(R)=\{\alpha\}$. Comme $c \in \lim _{R \rightarrow \infty} I(R)$, on obtient $c=\alpha$. Ainsi à chaque $L^{i}$ correspond au plus une valeur critique asymptotique. D'où $K_{\infty}(f)$ n'a qu'un nombre fini de points.

REMARQue 3.5. Le théorème 3.4 reste vrai pour une fonction définissable de classe $C^{1}$ définie sur une sous-variété de $\mathbb{R}^{n}$. Cette remarque est capitale pour la démonstration du théorème 4.2 , où l'on se réduit précisément à cette situation.

4. Valeurs critiques asymptotiques généralisées. On considère un ouvert $\Omega \subset \mathbb{R}^{n}$. On va montrer qu'il existe une métrique riemannienne qui rend $\Omega$ complet et telle que les valeurs critiques asymptotiques généralisées de toute fonction $f$ de classe $C^{2}$ forment un ensemble fini. Pour cela on va se ramener au cas du théorème 3.4 pour une fonction définie sur une sousvariété de $\mathbb{R}^{n+1}$. L'idée est d'utiliser une fonction tapissante $g$ de classe $C^{2}$, et d'étudier les valeurs critiques asymptotiques d'une fonction $\widetilde{f}$, difféomorphe à la fonction $f$, et définie sur le graphe de la restriction de la fonction $1 / g$ à l'ensemble $\Omega$. Rappelons un théorème de Palais (cf. [10] et [11]) qui s'exprime de la façon suivante: Soit $(\Omega, \delta)$ une variété riemannienne complète et soit $f: \Omega \rightarrow \mathbb{R}$ une fonction de classe $C^{2}$. Alors $f: \Omega \backslash f^{-1}\left(\mathcal{K}_{f}\right) \rightarrow \mathbb{R} \backslash \mathcal{K}_{f}$ est une fibration triviale au-dessus de chaque composante connexe de $\mathbb{R} \backslash \mathcal{K}_{f}$. 
Le théorème suivant assure l'existence de fonctions tapissantes dans les structures o-minimales :

ThÉORÈme 4.1 (van den Dries-Miller). Soit $\Omega$ un ouvert définissable de $\mathbb{R}^{n}$. Alors il existe une fonction $g$ définissable, de classe $C^{2}$, strictement positive sur $\Omega$ et nulle sur $\mathbb{R}^{n} \backslash \Omega$.

Preuve. Voir [3] pour l'existence d'une fonction de classe $C^{k}$, où $k$ est un entier arbitraire.

ThÉORÈme 4.2. Soit $\Omega$ un ouvert définissable inclus dans $\mathbb{R}^{n}$. Alors il existe une métrique riemannienne $\delta$ sur $\Omega$ telle que $(\Omega, \delta)$ est complet, et quelle que soit $f$ définissable et de classe $C^{2}$ sur $\Omega$, l'ensemble

$$
\mathcal{K}_{f}=\left\{c \in \mathbb{R}: \exists\left\{x_{p}\right\}_{p \in \mathbb{N}} \subset \Omega, \lim _{p \rightarrow \infty} f\left(x_{p}\right)=c, \lim _{p \rightarrow \infty}\left\|\nabla_{\delta} f\left(x_{p}\right)\right\|=0\right\}
$$

ne contient qu'un nombre fini de points. Ici $\nabla_{\delta}$ désigne le gradient associé à la métrique riemannienne $\delta$, et $\mathcal{K}_{f}=K_{0}(f) \cup K_{\infty}(f)$ où $K_{0}(f)$ désigne l'ensemble des valeurs critiques de la fonction $f$ et $K_{\infty}(f)$, l'ensemble $\left\{c \in \mathcal{K}\right.$ : aucune sous-suite $\left\{x_{p}\right\}_{p \in \mathbb{N}}$ ne converge dans $\left.\Omega\right\}$.

Pre uve. Notons une fois pour toutes que $K_{0}(f)$ est un ensemble fini. Il suffit seulement d'étudier l'ensemble $K_{\infty}(f)$. Soit $g$ une fonction définissable, vérifiant les propriétés du théoréme 4.1 pour l'ouvert $\Omega$, et soit $g_{\Omega}$ le graphe de la restriction à l'ensemble $\Omega$ de la fonction $1 / g$. Alors $g_{\Omega}$ est un ensemble définissable dans $\mathbb{R}^{n+1}$ et difféomorphe à $\Omega$. A chaque fonction $f$ vérifiant les hypothèses du théorème, on associe une fonction $\widetilde{f}$ sur $g_{\Omega}$, de sorte que pour $(x, 1 / g(x)) \in g_{\Omega}, \widetilde{f}(x, 1 / g(x))=f(x)$.

Soit $\mu$ une métrique sur $g_{\Omega}$ de sorte que le gradient associé $\nabla_{\mu}$ est tel que

$$
\left\|\nabla_{\mu} \widetilde{f}(y)\right\|=(1+\|y\|)\|\nabla \widetilde{f}(y)\|
$$

où $y \in g_{\Omega}$ et $\nabla$ désigne la restriction sur $g_{\Omega}$ du gradient associé à la métrique euclidienne. Notons que l'ensemble $g_{\Omega}$ étant localement une sous-variété de $\mathbb{R}^{n} \times \mathbb{R}$, il est complet pour la métrique $\mu$.

En vertue de la remarque 3.5 et du théorème $3.4, K_{\infty}(\widetilde{f})$ est réunion finie de points de $\mathbb{R}$.

Or l'application $\mathcal{G}=(\mathrm{Id}, 1 / g): \Omega \rightarrow g_{\Omega}$ étant un difféomorphisme, elle induit une isométrie entre $(\Omega, \delta)$ et $\left(g_{\Omega}, \mu\right)$, où $\delta$ vèrifie $\delta(u, v)=\mu\left(\mathcal{G}^{*}(u)\right.$, $\left.\mathcal{G}^{*}(v)\right)$ pour tous $u, v \in T \Omega$. Evidemment, $(\Omega, \delta)$ est complet, et $K_{\infty}(\widetilde{f}) \cup$ $K_{0}(\widetilde{f})=\mathcal{K}_{f}$. Or comme $K(\widetilde{f})$ et $K_{\infty}(\widetilde{f})$ sont des ensembles finis de points, alors $\mathcal{K}(f)$ l'est également, ce qui termine la démonstration.

5. Exemples et remarques. Soit $f$ une fonction définissable, de classe $C^{1}$, définie sur $\mathbb{R}^{n}$. Dans [8], Loi et Zaharia prennent comme définition de 
valeurs critiques asymptotiques pour la fonction $f$ l'ensemble

$$
\begin{aligned}
S_{f}=\left\{c \in \mathbb{R}: \exists\left(x_{p}, \lambda_{p}\right) \in \mathbb{R}^{n} \times \mathbb{R},\right. \\
\left.\qquad \lim _{p \rightarrow \infty}\left\|x_{p}\right\|=\infty, \lim _{p \rightarrow \infty} f\left(x_{p}\right)=c, \nabla f\left(x_{p}\right)=\lambda_{p} x_{p}\right\} .
\end{aligned}
$$

Notons que $\lambda_{p}$ est non nul à partir d'un certain rang.

ThÉORÈme 5.1. Soit $f$ comme ci-dessus. Alors $S_{f} \subseteq K_{\infty}(f)$.

Pr e u ve. Soit $c \in S_{f}$. Alors il existe une suite $\left\{x_{p}, \lambda_{p}\right\} \in \mathbb{R}^{n} \times \mathbb{R}$ vérifiant les conditions de la définition, et comme dans tout ce qui précède, il existe $R>0$ ainsi qu'une fonction $\lambda$ et un arc $\gamma$, tous deux définissables $C^{1}$ et définis sur $] R, \infty$ [, tels que $\lim _{r \rightarrow \infty} f(\gamma(r))=c, \lim _{r \rightarrow \infty}\|\gamma(r)\|=\infty$ et $\nabla f(\gamma(r))=\lambda(r) \gamma(r)$ pour tout $r \in] R, \infty[$.

Comme précedemment, on pose $h=f \circ \gamma$ et $\alpha(r)=\cos \left(\gamma(r), \gamma^{\prime}(r)\right)$. Il est facile de voir que

$$
\lambda(r)=\frac{h^{\prime}(r)}{\|\gamma(r)\| \cdot\left\|\gamma^{\prime}(r)\right\| \alpha(r)} .
$$

Et si on reparamétrise $\gamma$ pour que $\|\gamma(r)\|=r$, alors on a

$$
\|\gamma(r)\| \cdot\|\nabla f(\gamma(r))\|=\frac{1}{\left\|\gamma^{\prime}(r)\right\| \alpha(r)} r h^{\prime}(r) .
$$

De plus (cf. section 2), on a $\left\|\gamma^{\prime}(r)\right\| \alpha(r) \rightarrow 1$, et $r h^{\prime}(r) \rightarrow 0$ quand $r \rightarrow \infty$. Ainsi, $c \in K_{\infty}(f)$, ce qui termine la preuve.

Exemples ET REMARques 5.2. Il convient de remarquer que $S_{f}$ contient encore des points superflus, et ne discrimine pas totalement les points de bifurcations de la fonction $f$. Mais, bien que plus fin que $K_{\infty}(f)$, il ne semble pas être davantage exploitable pour caractériser plus précisément l'ensemble des bifurcations de fonctions définissables.

Dans les exemples qui suivent, on donne différentes configurations pour $S_{f}, K_{\infty}(f)$, et $B_{f}$ ( $B_{f}$ désigne les points de bifurcation). On s'est restreint à regarder des fonctions définies sur $\mathbb{R}^{2}$.

1. $f(x, y)=y /\left(1+x^{2}\right)$. Alors $S_{f}=B_{f}=\emptyset$ et $\{0\}=K_{\infty}(f)$.

2. $f(x, y)=y(x y-1)$. Alors $S_{f}=B_{f}=K_{\infty}(f)=\{0\}$.

3. $f(x, y)=y \exp 2 x+\exp x$ (cf. [8]). Alors $B_{f}=\emptyset$ et $S_{f}=K_{\infty}(f)=\{0\}$.

\section{Bibliographie}

[1] J. Bochnak, M. Coste and M. F. Roy, Real Algebraic Geometry, Ergeb. Math. Grenzgeb. 36, Springer, 1998.

[2] L. van den Dries, Tame Topology and o-Minimal Structures, London Math. Soc. Lecture Note Ser. 248, Cambridge Univ. Press, 1988. 
[3] L. van den Dries and C. Miller, Geometric categories and o-minimal structures, Duke Math. J. 84 (1996), 497-540.

[4] K. Kurdyka, On a subanalytic stratification satisfying a Whitney property with exponent 1, in: Real Algebraic Geometry (Rennes, 1991), Lecture Notes in Math. 1524, Springer, 1992, 316-322.

[5] - On gradients of functions definable in o-minimal structures, Ann. Inst. Fourier (Grenoble) 48 (1998), 769-783.

[6] K. Kurdyka, T. Mostowski and A. Parusiński, Gradient conjecture in ominimal structures, en préparation.

[7] K. Kurdyka, P. Orro and S. Simon, Semialgebraic Sard theorem for generalized critical values, preprint, Univ. Savoie, 1999.

[8] T. L. Loi and A. Zaharia, Bifurcation sets of functions definable in o-minimal structures, Illinois J. Math. 42 (1998), 449-457.

[9] C. Miller, Exponentiation is hard to avoid, Proc. Amer. Math. Soc. 122 (1994), 257-259.

[10] R. S. Palais, Lusternik-Schnirelman theory on Banach manifolds, Topology 5 (1966), 115-132.

[11] A. Parusiński, On the bifurcation set of complex polynomial with isolated singularities at infinity, Compositio Math. 97 (1995), 369-384.

[12] P. J. Rabier, Ehresmann fibrations and Palais-Smale conditions for morphisms of Finsler manifolds, Ann. of Math. 146 (1997), 647-691.

LAMA, Université de Savoie

73376 Le Bourget du Lac Cedex, France

E-mail: ddacu@univ-savoie.fr

Reçu par la Rédaction le 28.7.1999

Révisé le 10.2.2000 